\title{
A atenção aos cuidadores informais/familiares e a estratégia de Saúde da Família: contribuições de uma revisão bibliográfica*
}

\author{
Providing care for informal/family caregivers and \\ the Family Health strategy: contributions \\ of a Literature review
}

\author{
Marília Rafacho', Fátima Corrêa Oliver²
}

RAFACHO, M.; OLIVER, F. C. A atenção aos cuidadores informais/familiares e a Estratégia de Saúde da Família: contribuições de uma revisão bibliográfica. Rev. Ter. Ocup. Univ. São Paulo, v. 21, n. 1, p. 41-50, jan./abr. 2010.

\begin{abstract}
RESUMO: Trata-se de uma revisão bibliográfica sobre atenção aos cuidadores familiares na Estratégia de Saúde da Família (ESF) realizada por levantamento de artigos, dissertações, teses, livros, monografias, guias e resumos (período de 1997 a 2008) na literatura brasileira. Foram encontrados 242 textos sobre o tema, sendo $55 \%$ de artigos e 2005 o ano com maior número de publicações. A maioria dos textos foi produzida por profissionais de enfermagem e concentra-se em revistas e faculdades dessa área na região sudeste. Adotou-se 10 temáticas para classificação dos textos: percepção dos cuidadores sobre o cuidado prestado ao familiar; impacto/sobrecarga nos cuidadores; caracterização, acompanhamento de cuidadores por profissionais; cotidiano/ fazer do cuidador; estudo teórico; revisão bibliográfica; orientação aos cuidadores; cuidadores e sua experiência e outras temáticas. Foram encontrados apenas 20 textos no contexto do PSF, publicados entre 2003 e 2008, a maioria a respeito da percepção dos cuidadores sobre o cuidado diário ao familiar. Observou-se que se trata de um tema relativamente recente na literatura estudada, principalmente quando abordam essa questão na Atenção Básica e que há necessidade dos profissionais de se aproximarem e aprofundarem os estudos.
\end{abstract}

DESCRITORES: Cuidadores. Programa saúde da família. Saúde da família. Terapia ocupacional. Literatura de revisão como assunto.

\footnotetext{
* Este estudo é parte do processo de avaliação das disciplinas MFT0213 - Iniciação à Pesquisa I e MFT0254 - Iniciação à Pesquisa II Terapia Ocupacional e Saúde da Pessoa com Deficiência, do curso de graduação em Terapia Ocupacional do Departamento de Fisioterapia, Fonoaudiologia e Terapia Ocupacional da FMUSP.

1. Discente do curso de Terapia Ocupacional do Departamento de Fisioterapia, Fonoaudiologia e Terapia Ocupacional da FMUSP, no período de 2006-2010.

${ }^{2}$. Docente do Curso de Terapia Ocupacional do Departamento de Fisioterapia, Fonoaudiologia e Terapia Ocupacional da FMUSP.

Endereço para correspondência: Rua Cipotânea, 51 Cidade Universitária CEP 05360-160 São Paulo - SP. E-mail: fcoliver@usp.br
} 


\section{INTRODUÇÃO}

$\mathrm{O}$ estudo busca conhecer como a questão dos cuidadores familiares é discutida na literatura brasileira do campo da saúde e como se articula com a Estratégia de Saúde da Família (ESF).

O Programa de Saúde da Família (PSF), que deu origem à Estratégia de Saúde da Família, é uma proposta que reafirma os princípios de universalização, descentralização, integralidade e participação da comunidade e tem como principal objetivo criar condições favoráveis para mudança do modelo tradicional da prática assistencial centrada na doença e na hospitalização, desde a atenção básica em saúde. É uma estratégia assistencial e de organização de serviços nesse nível de atenção com foco no cuidado à família e na ação sobre os determinantes de saúde de uma dada população (AMENDOLA, 2007).

$\mathrm{O}$ atual perfil demográfico e epidemiológico brasileiro, caracterizado pelo envelhecimento populacional e aumento da incidência de doenças crônico-degenerativas, evidencia o aparecimento de grupos populacionais com limitações nas atividades cotidianas e maiores necessidades de cuidados à saúde. A família, enquanto principal responsável pela formação pessoal e social dos indivíduos, assume a função de cuidado diante de tais situações e seu papel torna-se particularmente relevante durante períodos transitórios ou permanentes de menor capacidade física e/ ou psíquica de seus membros (ALMEIDA, 2005).

Nesse contexto surge a figura do cuidador, pessoa que presta cuidados fundamentais a alguém que apresente algum tipo de dependência, de forma parcial ou integral. Para Karsch (2003), o termo se refere a uma pessoa que chama para si a incumbência de apoiar a pessoa que vivencia um episódio mórbido ou ainda aquelas com alguma limitação (cognitiva, sensorial ou física) na realização de determinadas tarefas, desde a higiene pessoal até a administração financeira da família.

O papel de cuidador pode ser informal, quando assumido por um membro da família ou da comunidade, e formal, quando assumido por um profissional com formação específica ou contratado. Nesta pesquisa foi considerado apenas o cuidador informal.

Segundo o Guia Prático do Cuidador, do Ministério da Saúde (2008), o papel do cuidador ultrapassa o simples acompanhamento das atividades diárias dos indivíduos e, na maioria dos casos, essa pessoa não possui preparo adequado ou suporte para lidar com tais cuidados, além disso, as atividades prestadas somam-se a outras cotidianas.
O cuidador tem um importante papel de ligação entre a equipe de saúde e a pessoa cuidada, executando tarefas recomendadas. Entretanto, mais que isso, como membro da família, o cuidador é usuário do serviço de saúde e também requer atenção específica, inclusive de caráter preventivo. A esse respeito, Araújo et al. (2009) apontam que o foco de atenção na prática profissional, na maioria das vezes, é o indivíduo doente, cabendo ao cuidador uma posição mais à margem dos acontecimentos.

Recentemente, no Brasil, a atenção básica em saúde conta com os Núcleos de Apoio à Saúde da Família (NASF), com os objetivos de ampliar a abrangência e escopo das ações da Atenção Básica (BRASIL, 2008) e apoiar a inserção da ESF na rede de serviços. Dessa forma, os NASF's devem atuar de modo integrado à rede de serviços do território, onde é implantado, para complementar e compartilhar as práticas das Equipes de Saúde da Família, por meio de uma equipe multiprofissional.

Considerando tais atribuições, um dos profissionais que adquire relevância na equipe é o terapeuta ocupacional. Sua prática pode envolver o conhecimento e contato direto com a dinâmica da família, propondo intervenções que influenciem nos processos saúde/doença de seus membros e da comunidade e, assim, auxiliar os cuidadores a lidarem com a sobrecarga emocional e ocupacional gerada pelo cuidado oferecido.

Considerando tais aspectos, cuidar de quem cuida "passa a ser então um problema real e uma função no papel dos profissionais de saúde, principalmente aqueles vinculados à Atenção Primária a Saúde" (ALMEIDA, 2005).

Este estudo tem como objetivo conhecer como a literatura brasileira em saúde tem tratado o tema cuidadores informais, com especial ênfase na atenção básica em saúde.

\section{MÉTODOS}

Trata-se de estudo de revisão bibliográfica de textos (leis, manuais, livros, artigos, entre outros) através de busca eletrônica de trabalhos divulgados na Scientific Eletronic Library Online (SciELO), na Literatura Latino-Americano em Ciências de Saúde (Lilacs), no Ministério da Saúde pela Biblioteca Virtual em Saúde (BVS) e no Dedalus, banco de dados bibliográfico da Universidade de São Paulo.

Definiu-se como critério de inclusão apenas textos nacionais, em português, publicados entre 1997 e 2008. Foram utilizadas as seguintes palavras chaves: Programa and saúde and família; Cuidadores; cuidadores and programa and saúde and família. 
Após localização das referências foi realizada a leitura dos respectivos resumos e, em seguida, foram analisados e selecionados os textos de interesse para o estudo.

Foi criado um banco de dados dos textos localizados com data de publicação; tipo de produção; formação profissional do autor; além dos grupos etários e tipos de doença e/ou incapacidade das pessoas cuidadas. A análise dos dados compreendeu a identificação do conteúdo dos textos localizados nas seguintes categorias:

- Percepção dos cuidadores: diz respeito ao modo como estes compreendem os cuidados prestados ao familiar dependente, seu papel como cuidador e o impacto da doença na família;

- Impacto/sobrecarga do trabalho dos cuidadores: refere-se ao impacto/sobrecarga gerados nos cuidadores devido às funções que realizam e às modificações no cotidiano implicadas pelo processo de cuidar;

- Caracterização dos cuidadores: aponta o perfil sócio-demográfico dos cuidadores e pacientes;

- Acompanhamento de cuidadores por profissionais: descreve e discute algum tipo de intervenção, programa ou projeto direcionado para este grupo;

- Cotidiano/fazer do cuidador: atividades realizadas diariamente pelos cuidadores, as mudanças do seu papel na família e as estratégias que elaboram para o enfrentamento de problemáticas relacionadas ao cuidar;

- Estudo teórico sobre cuidadores: textos que discutem aspectos relacionados ao cuidador e cuidados prestados, a partir de referenciais teóricos específicos;

- Revisão bibliográfica: verifica e analisa as publicações anteriores sobre o tema cuidadores;

- Orientação a cuidadores: textos de manuais, guias ou livros para nortear e auxiliar nas atividades de cuidado diário;

- Cuidadores e sua experiência: relatos de histórias vivenciadas no cuidar de uma pessoa dependente, narrados pelo próprio cuidador ou profissionais;

- Outras temáticas: textos que não se enquadram em nenhuma das temáticas anteriores.

\section{RESULTADOS E DISCUSSÃO}

Foram identificados 242 textos. Conforme apresentado no Gráfico 1, percebe-se um aumento de publicações entre 1997 e 2000 e nos anos 2004 e 2005, mas a partir de 2006 houve decréscimo do número de publicações sobre o tema, com leve aumento em 2008.

Ao analisar a distribuição por tipos de publicações, Gráfico 1, observa-se que há predomínio de artigos em todos os anos, representando mais de 55\% do total de publicações, a maioria no ano de 2005. Manuais e monografias representam apenas $2,1 \%$. O ano de menor produção de artigos coincide com o de maior produção das teses de doutorado, em 2001. As dissertações de mestrado demonstram ascensão entre 1999 e 2002 e acentuado declínio a partir de 2005.

Conforme a Tabela 1, os artigos encontram-se distribuídos em 61 periódicos relacionados à área da saúde, quase a metade $(48,2 \%)$ concentrada em revistas de enfermagem, demonstrando maior preocupação desse campo do conhecimento no estudo sobre o tema.

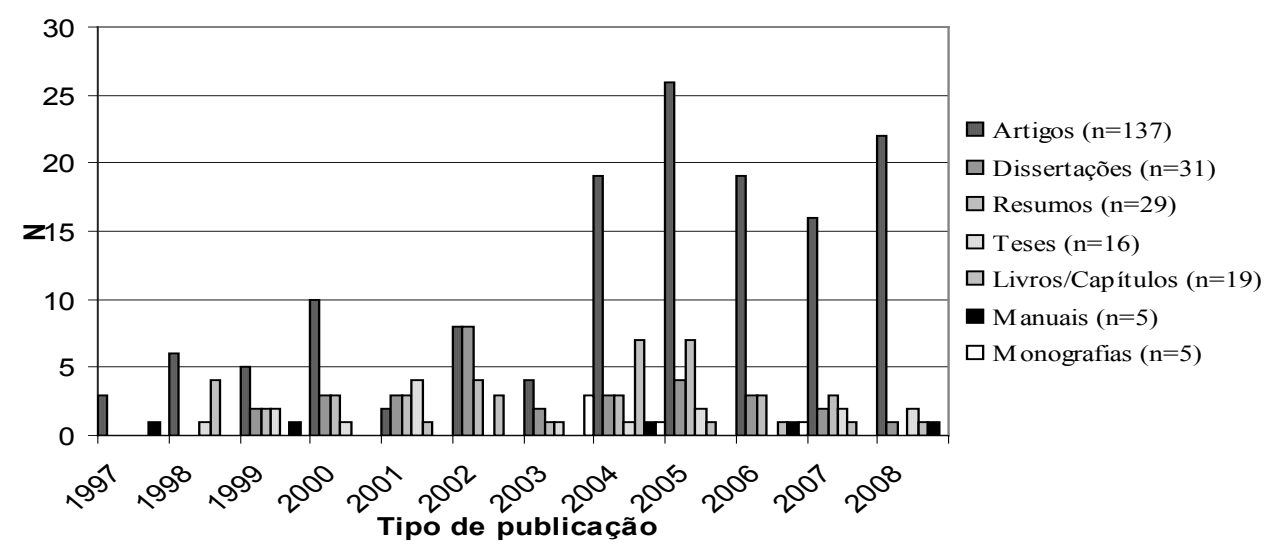

Gráfico 1 - Textos localizados, segundo tipo e ano de publicação 
RAFACHO, M.; OLIVER, F. C. A atenção aos cuidadores. Rev. Ter. Ocup. Univ. São Paulo, v. 21, n. 1, p. 41-50, jan./abr. 2010.

Tabela 1 - Distribuição dos artigos, segundo periódico de publicação

\begin{tabular}{|c|c|}
\hline Periódico & $\mathbf{n}$ \\
\hline Revista Brasileira de Enfermagem & 15 \\
\hline Texto e Contexto Enfermagem & 13 \\
\hline Revista da Escola de Enfermagem USP & 10 \\
\hline Revista O Mundo da Saúde & 8 \\
\hline Revista Mineira de Enfermagem & 6 \\
\hline Acta Paulista de Enfermagem & 5 \\
\hline Caderno de Saúde Pública & 5 \\
\hline Acta Scientiarum Health Sciences & 4 \\
\hline Revista Cogitare Enfermagem & 4 \\
\hline Revista Latino-Americana de Enfermagem & 4 \\
\hline Ciência e Saúde Coletiva & 3 \\
\hline Revista Gaúcha de Enfermagem & 3 \\
\hline Saúde e Sociedade & 3 \\
\hline Ciência Cuidado e Saúde & 2 \\
\hline Psicologia Estudantil & 2 \\
\hline Revista Brasileira de Cancerologia & 2 \\
\hline Revista Brasileira de Geriatria e Gerontologia & 2 \\
\hline Revista de Psiquiatria Clínica & 2 \\
\hline Textos sobre Envelhecimento & 2 \\
\hline Revista Arquivos Catarinense de Medicina & 1 \\
\hline Acta Fisiátrica & 1 \\
\hline Arquivos Brasileiros de Psicologia & 1 \\
\hline Arquivos Ciência e Saúde & 1 \\
\hline Arquivos de Geriatria e Gerontologia & 1 \\
\hline Arquivos de Neuropsiquiatria & 1 \\
\hline Escola Anna Nery Revista de Enfermagem & 1 \\
\hline Revista Espaço saúde & 1 \\
\hline Revista Imaginário & 1 \\
\hline Informação Psiquiátrica & 1 \\
\hline Interface (Botucatu) & 1 \\
\hline Jornal Brasileiro de Psiquiatria & 1 \\
\hline Journal Epilepsy Clinical Neurophysiol & 1 \\
\hline Jornao Pediátrico & 1 \\
\hline Online Brazilian Journal of Nursing & 1 \\
\hline Pediatria Moderna & 1 \\
\hline Physis & 1 \\
\hline Revista Psicologia Argumento & 1 \\
\hline Psicologia em Revista & 1 \\
\hline Revista Psicologia & 1 \\
\hline Psicologia: Reflexão e Crítica & 1 \\
\hline Psicologia: Teoria e Pesquisa & 1 \\
\hline Psicologia USP & 1 \\
\hline Revista Associaçẫo Médica Brasileira & 1 \\
\hline Revista de Psiquiatria Rio Grande Sul & 1 \\
\hline Revista Eletrônica de enfermagem & 1 \\
\hline Revista Baiana de Enfermagem & 1 \\
\hline Revista Brasileira de Crescimento e Desenvolvimento Humano & 1 \\
\hline Revista Brasileira de Cirurgia Cardiovascular & 1 \\
\hline Revista Brasileira de Ginecologia e Obstetrícia & 1 \\
\hline Revista Brasileira de Neurologia & 1 \\
\hline Revista Brasileira de Reumatologia & 1 \\
\hline Revista de Saúde Pública & 1 \\
\hline Revista Kairós & 1 \\
\hline Revista Paulista de Enfermagem & 1 \\
\hline Revista Sociedade Brasileira de Clínica Médica & 1 \\
\hline Revista SPAGESP & 1 \\
\hline Revista Rede de Enfermagem do Nordeste & 1 \\
\hline Revista Uningá & 1 \\
\hline Revista Saúde em debate & 1 \\
\hline Revista Scientia Médica & 1 \\
\hline Temas de desenvolvimento & 1 \\
\hline Total & 137 \\
\hline
\end{tabular}


RAFACHO, M.; OLIVER, F. C. A atenção aos cuidadores. Rev. Ter. Ocup. Univ. São Paulo, v. 21, n. 1, p. 41-50, jan./abr. 2010.

As dissertações e teses foram realizadas em sua maioria em instituições de ensino de enfermagem da região sudeste, principalmente no estado de São Paulo.

As temáticas mais prevalentes nos textos são apresentadas na Tabela 2, onde aqueles sobre a percepção dos cuidadores representam a maior parte das publicações $(19,4 \%)$, seguidos daqueles sobre o impacto/sobrecarga do cuidador e sua caracterização. Chama a atenção, o fato de textos de orientação aos cuidadores e de estudos sobre a experiência de cuidadores constituírem apenas 3,7\% e 2,1\% do total de publicações, sendo este último publicado somente até 2002. Artigos, dissertações e resumos acompanharam esta tendência, enquanto teses e monografias buscaram tratar de outras temáticas e livros/capítulos apresentaram predominância de estudos teóricos (Tabela 3).

Tabela 2 - Temáticas presentes nas publicações estudadas, segundo ano de publicação

\begin{tabular}{|c|c|c|c|c|c|c|c|c|c|c|c|c|c|c|}
\hline Temáticas & 1997 & 1998 & 1999 & 2000 & 2001 & 2002 & 2003 & 2004 & 2005 & 2006 & 2007 & 2008 & \multicolumn{2}{|c|}{ Total } \\
\hline & & & & & & & & & & & & & $n$ & $\%$ \\
\hline $\begin{array}{l}\text { Percepção dos } \\
\text { cuidadores }\end{array}$ & - & 1 & - & 2 & 2 & 5 & 2 & 7 & 12 & 4 & 5 & 7 & 47 & 19,4 \\
\hline $\begin{array}{l}\text { Impacto/sobrecarga } \\
\text { do trabalho }\end{array}$ & - & 4 & 1 & 2 & 2 & 3 & 1 & 7 & 9 & 3 & 6 & 4 & 42 & 17,4 \\
\hline $\begin{array}{l}\text { Caracterização dos } \\
\text { cuidadores }\end{array}$ & 1 & 1 & 1 & 3 & 1 & 2 & 2 & 1 & 5 & 7 & 5 & 4 & 33 & 13,6 \\
\hline $\begin{array}{l}\text { Acompanhamento } \\
\text { de cuidadores }\end{array}$ & - & - & 1 & 4 & - & 7 & 1 & 4 & 5 & 5 & 1 & 2 & 30 & 12,4 \\
\hline $\begin{array}{l}\text { Cotidianolfazer do } \\
\text { cuidador }\end{array}$ & - & 1 & 4 & 2 & 5 & 2 & - & 3 & 3 & 2 & 1 & 2 & 25 & 10,3 \\
\hline Outras temáticas & 1 & 1 & 1 & 1 & 2 & 3 & 2 & 3 & 1 & 2 & 4 & 1 & 22 & 9,1 \\
\hline $\begin{array}{l}\text { Revisão } \\
\text { bibliográfica }\end{array}$ & - & - & 2 & - & - & - & - & 4 & 4 & 2 & 1 & 4 & 17 & 7,0 \\
\hline $\begin{array}{l}\text { Estudo teórico } \\
\text { sobre cuidadores }\end{array}$ & - & - & - & 1 & 1 & - & 2 & 4 & - & 1 & 1 & 2 & 12 & 5,0 \\
\hline $\begin{array}{l}\text { Orientação a } \\
\text { cuidadores }\end{array}$ & 1 & 1 & 1 & - & - & 1 & - & 2 & 1 & 1 & - & 1 & 9 & 3,7 \\
\hline $\begin{array}{l}\text { Cuidadores e sua } \\
\text { experiência }\end{array}$ & 1 & - & 1 & 2 & - & - & 1 & - & - & - & - & - & 5 & 2,1 \\
\hline Total & 4 & 9 & 12 & 17 & 13 & 23 & 11 & 35 & 40 & 27 & 24 & 27 & 242 & 100 \\
\hline
\end{tabular}

Os textos foram publicados em sua maioria por profissionais da enfermagem $(59,1 \%)$, psicologia $(16,5 \%)$ e medicina $(9,9 \%)$. Apenas 6 publicações foram realizadas por terapeutas ocupacionais, dois artigos, dois resumos, uma dissertação e uma tese.

O campo da enfermagem apresentou principalmente temas sobre a caracterização e percepção do cuidador sobre o cuidado e sobre seu cotidiano/fazer, enquanto o da medicina tratou do impacto/sobrecarga dessa atividade, revisão bibliográfica e do trabalho de profissionais com cuidadores. Nos campos da psicologia e terapia ocupacional foram tratados a percepção do cuidador sobre o cuidado, o impacto/sobrecarga da atividade e o trabalho de profissionais com cuidadores.

A maior presença dessas temáticas pode indicar a necessidade dos profissionais de se aproximarem do tema por meio de pesquisas de campo; de esboçarem o perfil do cuidador no contexto de morbi-mortalidade brasileiro; de compreenderem suas crenças e valores sobre o cuidado, a saúde e a doença; de conhecerem as necessidades de cuidado e educação específicas para este grupo, além de identificarem o impacto e as alterações na qualidade de vida, devidos à rotina diária desse grupo. 
RAFACHO, M.; OLIVER, F. C. A atenção aos cuidadores. Rev. Ter. Ocup. Univ. São Paulo, v. 21, n. 1, p. 41-50, jan./abr. 2010.

Tabela 3 - Temáticas presentes nas publicações estudadas, segundo tipo de publicação

\begin{tabular}{|c|c|c|c|c|c|c|c|c|c|}
\hline \multirow[t]{2}{*}{ Temáticas } & \multirow[t]{2}{*}{ Artigos } & \multirow[t]{2}{*}{ Dissertações } & \multirow[t]{2}{*}{ Resumos } & \multirow[t]{2}{*}{ Teses } & \multirow{2}{*}{$\begin{array}{l}\text { Livrosl } \\
\text { Capitulos }\end{array}$} & \multirow[t]{2}{*}{ Manuais } & \multirow[t]{2}{*}{ Monografias } & \multicolumn{2}{|c|}{ Total } \\
\hline & & & & & & & & $n$ & $\%$ \\
\hline $\begin{array}{l}\text { Percepção dos } \\
\text { cuidadores }\end{array}$ & 31 & 10 & 3 & 2 & - & - & 1 & 47 & 19,4 \\
\hline $\begin{array}{l}\text { Impactolsobrecarga } \\
\text { do trabalho }\end{array}$ & 25 & 6 & 6 & 3 & 2 & - & - & 42 & 17,4 \\
\hline $\begin{array}{l}\text { Caracterização dos } \\
\text { cuidadores }\end{array}$ & 22 & 4 & 4 & 1 & 1 & - & 1 & 33 & 13,6 \\
\hline $\begin{array}{l}\text { Acompanhamento } \\
\text { de cuidadores }\end{array}$ & 16 & 1 & 6 & 3 & 3 & - & 1 & 30 & 12,4 \\
\hline $\begin{array}{l}\text { Cotidianolfazer do } \\
\text { cuidador }\end{array}$ & 12 & 7 & 2 & 2 & 2 & - & - & 25 & 10,3 \\
\hline Outras temáticas & 8 & 1 & 5 & 4 & 2 & - & 2 & 22 & 9,1 \\
\hline $\begin{array}{l}\text { Revisão } \\
\text { bibliográfica }\end{array}$ & 13 & 1 & 2 & - & 1 & - & - & 17 & 7,0 \\
\hline $\begin{array}{l}\text { Estudo teórico } \\
\text { sobre cuidadores }\end{array}$ & 7 & - & - & - & 5 & - & - & 12 & 5,0 \\
\hline $\begin{array}{l}\text { Orientação a } \\
\text { cuidadores }\end{array}$ & 1 & - & - & - & 3 & 5 & - & 9 & 3,7 \\
\hline $\begin{array}{l}\text { Cuidadores e sua } \\
\text { experiência }\end{array}$ & 2 & 1 & 1 & 1 & - & - & - & 5 & 2,1 \\
\hline Total & 137 & 31 & 29 & 16 & 19 & 5 & 5 & 242 & 100 \\
\hline
\end{tabular}

\section{Sobre as revisões bibliográficas acerca do tema cuidadores}

Foram encontrados 17 trabalhos de revisão bibliográfica no período de 1999 a 2008, sendo quatorze artigos, um resumo de evento e duas dissertações de mestrado.

Entre esses trabalhos, nove abordam o tema impacto/sobrecarga dos cuidadores, diferenciando-se pela característica clínica da pessoa cuidada: pessoas com demência elou doença de Alzheimer (GARRIDO; ALMEIDA, 1999; CRUZ; HAMDAN, 2008); pessoas que sofreram Acidente Vascular Cerebral (BOCCHI, 2004); pacientes psiquiátricos (BANDEIRA; BARROSO, 2005), idosos acamados (BRAZ; CIOSAK, 2005), crianças com autismo (FÁVERO; SANTOS, 2005); idosos com câncer (FLORIANI, 2004a; FLORIANI; SCHRAMM, 2006). Ainda, Amendola et al. (2005), realizaram levantamento bibliográfico acerca da qualidade de vida de cuidadores familiares.

Outro tema de revisão bibliográfica foi o papel e o fazer do cuidado: cuidado em família no contexto do domicílio (SENA et al.,1999); ato de cuidar para o familiar com ênfase na reabilitação psicossocial (VIANNA; BARROS, 2004); cotidiano do cuidador familiar e o processo de cuidar do idoso (MOREIRA; CALDAS, 2007; BRAZ; CIOSAK, 2008; NASCIMENTO et al., 2008). Ainda, Floriani (2004b), realizou outra revisão sobre cuidadores de idosos com câncer avançado e os aspectos bioéticos envolvidos em suas atividades.

Foram localizadas duas revisões sobre o trabalho com cuidadores de pessoas com demencia (LOUZADA; LOPES, 2004; LEME, 2006). Pegoraro e Caldana (2008) realizaram uma revisão na literatura nacional e estrangeira sobre a saúde mental da mulher enquanto possível provedora ou receptora de cuidados.

\section{Sobre as publicações no contexto da Estratégia de Saúde da Família}

Foram encontrados apenas 20 trabalhos sobre o tema, publicados a partir do ano de 2003, sendo a maioria 
em 2006 e 2007, onde mais da metade (65\%) refere-se a pesquisas realizadas em Unidades de Saúde da Família e em seus territórios de abrangência.

Há trabalhos publicados no período de 2003 a 2008, quase dez anos após a criação do PSF: treze artigos, quatro dissertações de mestrado, dois resumos e uma monografia de conclusão de curso. A formação dos autores variou entre medicina, fisioterapia, psicologia, terapia ocupacional e enfermagem, predominantemente esta última.

A exemplo do que ocorre nas publicações em geral, aquelas sobre a ESF também concentram-se na percepção dos cuidadores sobre o cuidado, seguidas pelas sobre sua caracterização (Tabela 4). Chama a atenção o fato de somente um trabalho abordar o acompanhamento de cuidadores por profissionais no contexto da ESF, onde Yamada (2006) et al. propõem uma reflexão bioética para profissionais e familiares envolvidos no cuidado a idosos dependentes, na área do PSF na cidade de Londrina, PR. Os autores concluem que, o cuidado ao idoso tem sido compreendido como uma responsabilidade exclusiva da família com pouca oferta ou inexistência de políticas públicas de proteção para esta população.

Tabela 4 - Temáticas presentes em publicações no contexto do PSF

\begin{tabular}{lccccccc}
\hline Temáticas & 2003 & 2004 & 2005 & 2006 & 2007 & 2008 & Total \\
\hline Percepçắo dos cuidadores & - & 1 & 1 & - & 3 & 2 & 7 \\
Impacto/sobrecarga & 1 & - & - & - & 1 & 1 & 3 \\
Caracterizaçä́o dos cuidadores & 1 & - & 1 & 3 & 1 & - & 6 \\
Acompanhamento de cuidadores & - & - & - & 1 & - & - & 1 \\
Cotidianoffazer do cuidador & - & - & - & 1 & - & - & 1 \\
Outras temáticas & - & - & - & 1 & 1 & - & 2 \\
Estudo teórico & - & - & - & - & - & - & - \\
Revisä́o bibliográfica & - & - & - & - & - & - & - \\
Orientaçä́o a cuidadores & - & - & - & - & - & - & - \\
Cuidadores e sua experiência & - & - & - & - & - & - & - \\
\hline Total & 2 & 1 & 2 & 6 & 6 & 3 & 20 \\
\hline
\end{tabular}

Abordando a temática percepção dos cuidadores, Morais (2004) apresentou um artigo de dissertação de mestrado sobre a representação da capacidade de realizar as tarefas de cuidado instrumental e qualidade de vida do cuidador de idosos dependentes no contexto do PSF de Uberaba-MG a partir da observação de aspectos psicossociais. Ainda sobre este assunto, Garcia et al. (2005), publicaram um estudo com um grupo de idosos e seus cuidadores sob atenção domiciliar em Unidades Básicas e de Saúde da Família de Campinas-SP, onde são descritas as percepções sobre as necessidades e dificuldades referentes ao cuidado e estado de interdependência e, em especial, aos aspectos emocionais e à qualidade de vida dos mesmos. Esses textos constataram deficiência do PSF como sistema de apoio às ações de saúde.

Ferreira (2007) estudou as representações sociais dos cuidadores de pessoas com HIVIAIDS sobre a doença e as possibilidades de seu enfrentamento, sobretudo no contexto doméstico com resultados que apontam sobrecarga associada ao cuidado, bem como os efeitos da discriminação em relação à contaminação de familiares pelo HIV.

Severo et al. (2007), em artigo sobre a vivência de familiares em relação ao portador de transtorno psíquico das áreas de cobertura do PSF em Natal-RN, reconhecem discursos de incapacidade diante da situação vivenciada e sentimento de insatisfação com os tratamentos recebidos pelo paciente. Machado et al. (2007), abordaram o cuidado que a família de portadores da Síndrome Metabólica de uma Unidade Básica de Saúde da Família de Fortaleza-CE assume no domicílio, a partir dos significados e crenças atribuídos pelos mesmos. São ressaltados tópicos sobre a percepção de si como cuidador, dificuldades estruturais vivenciadas no cuidado, significado da experiência de cuidar, mecanismos de enfrentamento, sentimento de despreparo para o cuidado entre outros.

Rocha et al. (2008) em estudo sobre o modo como os cuidadores da área adstrita de uma equipe do PSF em Montes Claros-MG interpretam e constroem o cotidiano identificaram situações adversas percebidas pelos cuidadores, tais como alterações físicas e de comportamento do idoso e falta de assistência para atender a suas necessidades e da pessoa cuidada. Mendonça et al. (2008), buscaram analisar o significado e as implicações de se tornar cuidador de um indivíduo com seqüelas de Acidente Vascular Encefálico (AVE) em uma Unidade de Saúde da Família (USF) em Londrina/PR e observaram ambivalência de sentimentos dos cuidadores, concluindo que cuidar de um familiar é uma tarefa complexa, que gera nos mesmos distintas formas de compreensão do cuidado.

Foram publicados três trabalhos a respeito do impacto ou sobrecarga dos cuidadores no PSF, nos domínios físico, psicológico, de relações sociais e meio ambiente e na sua qualidade de vida. Amendola (2003), em monografia de conclusão de curso avaliou a qualidade de vida de cuidadores domiciliares de pacientes com perdas funcionais e dependentes atendidos por uma equipe do PSF. A mesma autora, em dissertação de mestrado (AMENDOLA, 2007), e em artigo (AMENDOLA, 2008), abordou tema semelhante com famílias da região sul do município de São Paulo e considerou que o PSF pode ser instrumento para atender a necessidades de saúde de pacientes e seus cuidadores, contudo, a assistência prestada pelas Equipes de Saúde da Família é limitada. 
RAFACHO, M.; OLIVER, F. C. A atenção aos cuidadores. Rev. Ter. Ocup. Univ. São Paulo, v. 21, n. 1, p. 41-50, jan./abr. 2010.

Sobre a temática caracterização dos cuidadores, Mendes e Camargo (2003), apresentaram no I Curso de Especialização em Saúde da Família um estudo sobre a caracterização de idosos e cuidadores familiares atendidos no PSF de Icaraí-Ibaté, SP. Em dissertação, Almeida (2005), abordou a caracterização de cuidadores de idosos dependentes no contexto da saúde da família do Centro de Saúde Escola da Faculdade de Medicina de Ribeirão Preto, SP. Além do perfil dos cuidadores, apresentou as atividades básicas domésticas e de cuidado e o grau de dificuldade encontrado por estes.

Em 2006 foram publicados três artigos semelhantes que buscaram traçar o perfil de cuidadores familiares de idosos no PSF em diferentes estados. Gonçalves et al. (2006), caracterizaram as famílias cuidadoras de unidades básicas de saúde com ESF do município de Florianópolis, SC, entre 2004 e 2005. Enquanto, Vilela et al. (2006), descreveram cuidadores selecionados em todas as Unidades Básicas de Saúde com PSF do município de Jequié, BA, entre 2003 e 2004. Já Trelha et al. (2006), descreveram o perfil de idosos restritos ao domicílio e seus cuidadores assistidos por equipes do PSF de dois bairros em Londrina, PR. Todos os trabalhos apontaram a mulher de meia idade como principal cuidadora, casada e com apenas o ensino fundamental completo e diferenciaram-se apenas no que diz respeito às atividades extradomiciliares, onde apenas o último apresentou predominância de cuidadores com atividades fora do domicílio.

Ainda, Braz e Ciosak (2007), em resumo sobre a caracterização dos cuidadores senescentes de idosos dependentes, assistidos pelo PSF e pelo Programa de Assistência e Internação Domiciliar (PAID) do município de Cascavel-PR, consideraram que, os cuidadores senescentes enfrentam alterações em seu processo de envelhecimento e de saúde tornando-se tão ou mais vulneráveis do que aqueles que assistem.

Outra temática encontrada diz respeito à educação em saúde para cuidadores familiares. Martins et al. (2006); (2007), buscaram identificar as necessidades de educação em saúde para cuidadores leigos de idosos no domicílio, na área de abrangência da equipe do PSF do Município de São José, SC. Constataram que há uma deficiência na educação em saúde para o cuidador, carência de suporte e de recursos para prestação efetiva do cuidado ao idoso.

Acerca do cotidiano de cuidadores, Gonçalves et al. (2006), publicaram um artigo sobre o trabalho de cuidadoras no domicílio, identificadas por mapeamentos realizados pelas Equipes de Saúde da Família em Contagem, MG. Neste estudo, os autores destacam o cotidiano da mulher como principal cuidadora domiciliar, o que revela um fazer solitário e com pouco reconhecimento social e para o qual a rede de apoio é provisória e com insuficiente capacidade de atender às carências constantes.

A produção bibliográfica dos terapeutas ocupacionais tem tratado sobre o trabalho de profissionais com o cuidador (MATSUKURA et al., 2000; NOVELLI, 2005), a sua caracterização (NOVELLI, 2001), a sua percepção sobre o cuidado diário (MORAIS, 2004) e o impacto/sobrecarga dessa atividade (MATSUKURA, 2001; SOARES; CAMPOS, 2005).

\section{CONSIDERAÇÕES FINAIS}

A problemática do cuidador informal é campo de interesse e de estudos, principalmente na área de enfermagem e de relevância, em especial, para a Atenção Básica via ESF.

Ainda há poucas pesquisas que abordam os cuidadores na Atenção Básica, predominando os estudos sobre pacientes e cuidadores atendidos por serviços de assistência domiciliária vinculados a hospitais ou ainda que avaliam as necessidades do cuidador em relação à doença do paciente. A ESF, cenário em algumas pesquisas, é reconhecida como forma de atender às necessidades de saúde de pacientes e seus cuidadores considerando suas atribuições na atenção domiciliar. Entretanto, constatou-se que, mesmo em suas regiões de abrangência, a assistência prestada pelas Equipes de Saúde da Família mostrou-se limitada e focada no paciente, com carência de suporte e de recursos para prestação efetiva de acompanhamento e apoio aos cuidadores.

Nesse sentido, a questão dos cuidadores torna-se um tema de relevância e deve ter sua discussão aprofundada na implantação dos NASF's, que têm como principal objetivo apoiar e complementar as práticas realizadas pelas Equipes de Saúde da Família.

Muitas vezes, durante a atuação na comunidade, as demandas do cuidador se sobressaem às da pessoa cuidada, carecendo de uma intervenção mais contundente. Os estudos e as pesquisas sobre o tema são fundamentais para o reconhecimento de demandas dos cuidadores e contribuem com as Equipes de Saúde da Família, que poderão assistir o cuidador familiar em suas necessidades individuais, bem como considerá-los como um grupo específico, sujeitos e atores em ações de saúde.

Tendo em vista as demandas de atenção à saúde, colocadas pelo perfil demográfico e de morbimortalidade da população no Brasil, há necessidade de profissionais preparados para acompanhar demandas de saúde diferenciadas, o que justifica também a ampliação da pesquisa sobre o tema no Sistema Único de Saúde. 
RAFACHO, M.; OLIVER, F. C. A atenção aos cuidadores. Rev. Ter. Ocup. Univ. São Paulo, v. 21, n. 1, p. 41-50, jan./abr. 2010.

RAFACHO, M.; OLIVER, F. C. Providing care for informal/family caregivers and the Family Health Strategy: Contributions of a Literature Review. Rev. Ter. Ocup. Univ. São Paulo, v. 21 , n. 1, p. 41-50, jan./abr. 2010.

\begin{abstract}
This is a literature review on the care provided for family caregivers in the Family Health Program (PSF), based on articles, dissertations, theses, books, monographs, guides and abstracts (from 1997 to 2008) in the Brazilian literature. We found 242 texts on the theme, 55\% of which were articles. The year 2005had most publications. Most of the texts were produced by nursing professionals and were concentrated in their journals and higher education institutions in Brazilian's southeastern region. Texts were classified according to 10 themes: caregiver's perception about the care provided to the family, impact/burden on caregivers, characterization and monitoring of caregivers by professionals, caregiver's routine/activities, theoretical study, literature review, guidance to caregivers, caregivers and their experiences and other themes. In the literature published between 2003 and 2008 only 20 texts were found on the theme of PSF, most of which were on the caregivers perception about daily care, We noted that this is a relatively recent theme in the literature studied, especially with regards to Primary Care. There is need for professionals to approach and carry out further studies in this area.
\end{abstract}

KEY WORDS: Caregivers. Family health. Family health program. Occupational therapy. Review literature as topic.

\title{
REFERÊNCIAS
}

ALMEIDA, T. L. Características de cuidadores de idosos dependentes no contexto da saúde da família. 2005. Dissertação (mestrado - Enfermagem). Faculdade de Medicina de Ribeirão Preto, Univesidade de São Paulo, 2005.

AMENDOLA, F., et al. Como está sendo investigada e avaliada a qualidade de vida de cuidadores familiares. In: SOCIEDADE E SAÚDE. São Paulo, 2005. p. 301.

AMENDOLA, F. Qualidade de vida de cuidadores de pacientes com perdas funcionais e dependência atendidos em domicílio pelo programa de saúde da família do município de São Paulo. 2007. Dssertação (mestrado - Enfermagem). Escola de Enfermagem, Universidade de São Paulo, 2007.

AMENDOLA, F., et al. Qualidade de vida de cuidadores de pacientes dependentes no Programa de Saúde da Família. Texto Contexto Enferm., v. 17, n. 2, p. 266-272, 2008.

AMENDOLA, F. Qualidade de vida dos cuidadores de pacientes atendidos em domicilio por uma equipe de saude da familia da unidade básica Jardim Boa Vista. São Paulo: Escola de Enfermagem da Universidade de São Paulo, 2003.

ARAÚJO, L. Z. S., et al. Cuidador principal de paciente oncológico fora de possibilidade de cura, repercussões deste encargo. Rev. Bras. Enferm., v. 6, n. 1, p. 32-37, 2009.

BANDEIRA, M.; BARROSO, S. M. Sobrecarga das famílias de pacientes psiquiátricos. J. Bras. Psiquiatr., v. 54, n. 1, p. 34-46, 2005.

BOCCHI, S. C. M. Vivenciando a sobrecarga ao vir-a-ser um cuidador familiar de pessoa com acidente vascular cerebral (AVC): análise do conhecimento. Rev. Latinoamer. Enferm., v. 12, n. 1, p. 115-121, 2004.

BRASIL. Ministério da Saúde. Guia prático do cuidador. Brasília, 2008.

BRASIL. Ministério da Saúde. Portaria n. 154 de 24 de janeiro de 2008. Dispõe sobre a criação dos Núcleos de Apoio a Saùde da Família. Brasília, 2008, 03 mar.; Seção 1:1.

BRAZ, E.; CIOSAK, S. I. Caracterização dos cuidadores senescentes do Município de Cascavel - PR [CD-Rom]. In: SIMPÓSIO INTERNACIONAL DE POLÍTICAS E PRÁTICAS EM SAÚDE COLETIVA NA PERSPECTIVA DA EENFEMAGEM, 1., São Paulo-SP, 2007.

BRAZ, E.; CIOSAK, S. I. O cotidiano de cuidadores familiares de idosos dependentes. Rev. Uningá, v. 16, p. 41-52, 2008.

BRAZ, E.; CIOSAK, S. I. Perdas cotidianas do ser cuidador durante o processo do cuidar do idoso acamado: uma revisão bibliográfica. In: SEMINÁRIO NACIONAL DO ESTADO E POLÍTICAS SOCIAIS NO BRASIL, 2., Cascavel, 2005.

CRUZ, M. N.; HAMDAN, A. C. O impacto da doença de Alzheimer no cuidador. Psicol. Estudo, v. 13, n. 2, p. 223-229, 2008.

FÁVERO, M. A. B.; SANTOS, M. A. Autismo infantil e estresse familiar: uma revisão sistemática da literatura. Psicol. Reflexão Crítica, v. 18, n. 2, p.358-369, 2005.

FERREIRA, M. S. F. Cuidadores domésticos de pessoas com HIV/ Aids : representações sobre a doença e o cuidado. 2007. Dissertação (mestrado em psicologia) - Faculdade de Filosofia, Ciências e 
RAFACHO, M.; OLIVER, F. C. A atenção aos cuidadores. Rev. Ter. Ocup. Univ. São Paulo, v. 21, n. 1, p. 41-50, jan./abr. 2010.

Letras de Ribeirão Preto, Universidade de São Paulo, Ribeirão Preto, 2007.

FLORIANI, C. A. Cuidador familiar: sobrecarga e proteção. Rev. Bras. Cancerol., v. 50, n. 4, p. 341-345, 2004 a.

FLORIANI, C. A. Cuidador do idoso com câncer avançado: uma abordagem bioética. Rio de Janeiro: Escola Nacional de Saúde Pública da Fundação Oswaldo Cruz, 2004b.

FLORIANI, C. A.; SCHRAMM, F. R. Cuidador do idoso com câncer avançado: um ator vulnerado. Cad. Saúde Pública, v. 22, n. 3, p. 527-534, 2006.

GARCIA, M. A. A.; et al. Idosos e cuidadores fragilizados? Mundo Saúde, v. 29, n. 4, p. 645-652, 2005.

GARRIDO, R.; ALMEIDA, O. P. Distúrbios de comportamento em pacientes com demência: impacto sobre a vida do cuidador. Arq. Neuropsiquiatr., v. 7, n. 2-B, p. 424-434, 1999.

GONÇALVES, A. M., et al. Cuidadora domiciliar: por que cuido? Rev. Min. Enferm., v. 9, n. 4, p. 315-320, 2005.

GONÇALVES, L. H. T.; et al. Perfil da família cuidadora de idoso doente/fragilizado do contexto sociocultural de Florianópolis, SC. Texto Contexto - Enferm., v. 15, n. 4, p. 570-577, 2006.

KARSCH, U. M. Idosos dependentes: famílias e cuidadores. Cad. Saúde Pública, v. 19, n. 3, p. 861-866, 2003.

LEME, M. D. Treinamento de cuidadores de idosos: impacto na sua qualidade de vida e saúde: revisão sistemática. 2006. Dissertação (mestrado - medicina). Faculdade de Medicina, Universidade de São Paulo.

LOUZADA, B. A.; LOPES, M. H. I. Educação para cuidadores de idosos com demência. Scientia Médica, v. 14, n. 1, p. 77-80, 2004.

MACHADO, A. L. G.; et al. O fazer do cuidador familiar: significados e crenças. Rev. Bras. Enferm., v. 60, n. 50, p. 530-534, 2007.

MARTINS, J. J.; et al. Educação em saúde para leigos no cuidado ao idoso no contexto domiciliar. ACM Arq. Catarin. Med., v. 35, n. 4, p. 56-63, 2006.

MARTINS, J. J.; et al. Necessidades de educação em saúde dos cuidadores de pessoas idosas no domicílio. Texto Contexto - Enferm. v. 16, n. 2 , p. $254-262,2007$.

MATSUKURA, T. S.; et al. A Importância da provisäo de suporte aos cuidadores de crianças portadoras de transtornos do desenvolvimento. Temas Desenvolv., v. 8, n. 48, p. 5-10, 2000.

MATSUKURA, T. S. Mães de crianças com necessidades especiais: stress e percepção de suporte social. 2001 (Doutorado em Saúde Mental) - Departamento de Neurologia, Psiquiatria e Psicologia Médica, Faculdade de Medicina de Ribeirão Preto da Universidade de São Paulo, Ribeirão Preto.

MENDES, M. M. R.; CAMARGO, L. L. A. L. Caracterização dos idosos e cuidadores familiares do Programa de Saúde da Família do Jardim Icaraí - SP. In: ALMEIDA, M. C. P. Coletânea de trabalhos do I Curso de Especialização em Saúde da Família. 2003.
MENDONÇA, F. F.; et al. Cuidador familiar de seqüelados de acidente vascular cerebral: significado e implicações. Physis, v. 18, n. 1, p. 143-158, 2008.

MORAIS, H. Representações sobre o cuidado e a qualidade de vida do cuidador de idosos dependentes no programa de saúde da família de Uberaba-MG. 2004. Dissertação (mestrado em saúde na comunidade) - Faculdade de Medicina de Ribeirão Preto, Universidade de São Paulo, Ribeirão Preto.

MOREIRA, M. D.; CALDAS, C. P. A importância do cuidador no contexto da saúde do idoso. Esc. Anna Nery Rev. Enferm., v. 11, n. 3, p. 520-525, 2007.

NASCIMENTO, L. C.; et al. Cuidador de idosos: conhecimento disponível na base de dados LILACS. Rev. Bras. Enferm., v. 61, n. 4, p. 514-517, 2008.

NOVELLI, M. M. P. C.; et al. Cuidadores de pacientes com demência: perfil socio-demografico e impacto diário. In: REUNIÃO DE PESQUISADORES EM DOENÇA DE ALZHEIMER E DESORDENS RELACIONADAS, 3., Rio de Janeiro, 2001. Arq. Neuropsiquiatr., v. 59. p. 3, 2001.

NOVELLI, M. M. P. C.; et al. Efetividade de intervenções em grupo para cuidadores/familiares de pacientes com demência leve a moderada - Resultados preliminares. In: V REUNIÃO DE PESQUISADORES EM DOENÇA DE ALZHEIMER E DESORDENS RELACIONADAS, 5., Ribeirão Preto, 2005. Arq. Neuropsiquiatr., p. 80, 2005.

PEGORARO, R. F.; CALDANA, R. H. L. Mulheres, loucura e cuidado: a condição da mulher na provisão e demanda por cuidados em saúde mental. Saúde Soc., v. 17, n. 2, p. 82-94, 2008.

ROCHA, M.; et al. Desvelando o cotidiano dos cuidadores informais de idosos. Rev. Bras. Enferm., v. 61, n. 6, p. 801-808, 2008.

SENA, R. R.; et al. O cuidado no domicílio: um desafio em construção. Cogitare Enferm., v. 2, p. 58-62, 1999.

SEVERO, K. L.; et al. A experiência de familiares no cuidado em saúde mental. Arq. Bras. Psicol., v. 59, n. 2, p. 143-155, 2007.

SOARES, C. B.; CAMPOS, P. H. F. Representação da sobrecarga familiar e adesão aos serviços alternativos em saúde mental. Psicol. Rev., v. 11, n. 18, p. 219-237, 2005

TRELHA, C. S.; et al. Caracterização de idosos restritos ao domicílio e seus cuidadores. Espaço Saúde, v. 8, n. 1, p. 20-27, 2006.

VIANNA, P. C. M.; BARROS, S. O significado do cuidado para a família na reabilitação psicossocial do doente mental: uma revisão teórica. Rev. Mineira Enferm., v. 8, n. 1, p. 223-228, 2004.

VILELA, A. B. A.; et al. Perfil do famililar cuidador de idoso doente e/ou fragilizado do contexto sociocultural de Jequié-BA. Rev. Bras. Geriatr. Gerontol., v. 9, n. 1, p. 55-69, 2006.

YAMADA, K. N.; et al. Aspectos éticos envolvidos na assistência a idosos dependentes e seus cuidadores. Mundo Saúde, v. 30, n. 4, p. 667-672, 2006. 